\title{
Strain variation in microalgal lipid production during mixotrophic growth with glycerol
}

\author{
Kiran Paranjape ${ }^{1}$, Gustavo B. Leite ${ }^{1}$, and Patrick C. Hallenbeck ${ }^{1,2^{*}}$ \\ ${ }^{1}$ Département de Microbiologie, Infectiologie et Immunologie, Université de Montréal, CP6128 \\ Succursale Centre-ville, Montréal, Québec, Canada H3C 3J7 \\ ${ }^{2}$ Life Sciences Research Center, Department of Biology \\ United States Air Force Academy \\ 2355 Faculty Drive, USAF Academy, Colorado 80840 \\ *Corresponding author; Tel : (514)343-6278 Fax : (514)343-5701 \\ E-mail address : patrick.hallenbeck@ umontreal.ca
}

Keywords: microalgae; mixotrophic growth; lipid production; biofuels

Algal cultivation at high latitudes is challenged by the relatively low annual solar flux. One possible scenario to overcome this limitation is the use of mixotrophic growth to potentially boost biomass and lipid production. Here the effect of glycerol addition on the growth and lipid production by twelve indigenous microalgae was examined. The results show that there is considerable strain dependent variation in the maximum growth rate under mixotrophic conditions with the addition of glycerol causing in some cases up to a 2.4 fold increase in growth rate and a up to a 1.9 fold increase in biomass. In addition, glycerol increased total lipid production $40-60 \%$ in some strains. These results also show the value in screening culture collections for desired traits independent of strain identification since here one (PCH02) of the five Chlorella strains showed a large increase in lipid with glycerol. 


\section{Introduction}

The production of biofuels using microalgae has attracted a great deal of interest over the past decade with intense R\&D underway in a variety of areas including cultivation, harvesting and conversion into a useful fuel. There are outstanding challenges in each of these areas (Abdelaziz et al., 2013a,b; Christi 2013; Leite and Hallenbeck, 2012; Leite et al. 2013). Algal biofuels production in temperate regions faces additional challenges, in particular those due to climate (low temperatures) and geography, as these regions experience short days in the winter and, in general, reduced solar flux throughout the year, compared to tropical regions. Although some of these challenges, in particular temperature, can possibly be at least partially overcome through the selection of indigenous strains that are able to cope with this challenge (Abdelaziz et al., 2014; Hallenbeck et al., 2015), other strategies may be required to compensate for the lack of solar flux, a critical factor in constraining algal productivities.

One possible solution is to use mixotrophic growth, suppling fixed carbon to effectively supplement the autotrophic carbon dioxide fixation process compromised by the reduction in available light. Of course, this carbon was previously fixed through some type of plant photosynthesis, so such a process only makes sense if the fixed carbon substrate that is used comes from a readily available high volume waste stream. A number of recent reviews have examined the various waste streams and processes that might be involved (Dubey et al., 2015; Lowrey et al., 2015; Mitra et al., 2012; Mohan et al., 2015). Previous studies have shown that microalgae are, depending upon the species, capable of utilizing a variety of fixed carbon sources (Wang et al., 2014). However, the vast majority of these studies examined either hexoses, in particular glucose, or acetate, and it is difficult to see how these, especially glucose, would ever become available for practical application to algal cultivation. In particular, a glucose stream 
would more likely be fermented to bioethanol, a well-known industrial process with a conversion efficiency approaching 80-90\%.

Suitable carbon sources might be glycerol, available in large quantities $\left(>10^{9} \mathrm{~kg} / \mathrm{y}\right)$ as it is a by-product of biodiesel manufacture, or xylose, either available in the near future in large quantities as lignocellulosic conversion comes online, or now, in select waste streams such as the black liquor of the pulp and paper industry (Leite et al., 2015, Miazek et al., 2014). The use of such wastes in an integrated system can potentially greatly improve the LCA (life cycle assessment, as recently shown in a study applying such a system to bioethanol from sugar cane production where both the life cycle greenhouse gas emissions and the fossil energy use were predicted to be reduced (Souza et al., 2015).

Although not nearly as well studied as photoautotrophic growth, some research has previously been carried out on the heterotrophic or mixotrophic growth of microalgae (Chen and Walker, 2011; Koller et al., 2012; Ogawa and Aiba 1989; O’Grady and Morgan, 2011; Wang et al. 2014). As pointed out here, for economic reasons it is important to use cheap carbon sources, such as glycerol and a number of studies have examined mixotrophic growth of different species supported by glycerol. Thus, addition of glycerol $\left(5 \mathrm{~g} \mathrm{~L}^{-1}\right)$ increased both biomass productivities and the TAG content of Neochloris oleabundans, Botryococcus Braunii and Dunaliella sp. (Choi and Lee 2015). Likewise, the addition of glycerol into the growth medium has been reported to increase the total biomass production ( 2 fold) and lipid productivity of Scenedesmus sp. (Pancha et al., 2015). While most studies have examined the simple effect of adding glycerol on biomass and lipid production, in one study (Muto et al., 2014), the effects of glycerol were increased through the overexpression of the endogenous glycerol kinase of the oleaginous marine diatom

\section{Fistulifera solaris.}


A number of studies have examined mixotrophic growth with glycerol and/or lipid production with Chlorella with varying results. One study demonstrated that addition of glycerol actually inhibited the growth of Chlorella vulgaris (Heredia-Arroyo et al., 2011). Mixotrophic growth of Chlorella vulgaris with glycerol versus photoautotrophic growth has been reported to boost total lipid productivity, not through an increase in lipid content which actually decreased slightly, but through a large effect on biomass productivity which increased 10 fold, from $10 \mathrm{mg} \mathrm{L}^{-1} \mathrm{~d}^{-1}$ to 102 $\mathrm{mg} \mathrm{L}^{-1} \mathrm{~d}^{-1}$ when glycerol was present (Liang et al. 2009). Similar results have recently been noted with a newly isolated Chlorella sp. (Lin and Wu, 2015). Another study examining mixotrophic growth with glycerol found that Chlorella showed little difference in biomass concentration (only $17 \%$ increase 1.58 to $1.92 \mathrm{~g} \mathrm{~L}^{-1}$ ) at the optimal glycerol concentration $5 \mathrm{~g} \mathrm{~L}^{-}$ ${ }^{1}$, and although the lipid content increased markedly, more than four-fold, photoautotrophically grown cultures had a quite low lipid content (3.7\%) (Andruleviciute et al., 2014). The addition of technical glycerol $\left(2 \mathrm{~g} \mathrm{~L}^{-1}\right)$ improved the growth of Chlorella by $18 \%$ relative to the BG11 medium, reaching a biomass concentration of $1.92 \mathrm{~g} \mathrm{~L}^{-1}$ with a maximal biomass productivity of $0.172 \mathrm{~g} \mathrm{~L}^{-1} \mathrm{~d}^{-1}$ (Skorupskaite et al., 2015). Significantly, the biomass productivity of a Chlorella sorokiniana was greatly increased when grown on waste water amended with glycerol (Park et al., 2012). Finally, one study suggested the use of two-stage cultivation with an initial period of photoautotrophic growth followed by addition of glycerol (Yen and Chang, 2013).

Given the large variation in response by different species, and even different strains of Chlorella it was interesting to examine the effect of mixotrophic cultivation with glycerol on biomass and lipid production using some of the strains in the recently established collection of Quebec microalgae as this could potentially be used as a strategy to increase biofuels production in a solar flux limited locale. In a previous study (Leite et al., 2015) an examination of strains 
from this collection showed that at least some were capable of glycerol and xylose utilization for increased biomass and lipid production in the light. Here these findings have been extended to a larger number of strains. We hypothesized that some of the differences between these strains, and some of the apparently contradictory results previously obtained, were due to strain differences. In the present study, questions as to variation with species and strain have been examined by carrying out a rigorous phylogenetic identification. This allowed the demonstration that even among strains identified as Chlorella there is a wide variation in the response to glycerol, thus explaining some of the seeming contradiction between previous studies.

\section{Materials and Methods}

\subsection{Algal Cultivation}

The twelve algal strains used in this study were from the collection of the Laboratory of Advanced Biofuels, Département de microbiologie, infectiologie et immunology of the Université de Montréal. These strains were isolated in the region of Québec, Canada, and were previously described (Abdelaziz et al., 2014, Hallenbeck et al., 2014)). Bold's Basal medium (BBM) (Andersen, 2005) was used for photoautotrophic cultivation, and with $25 \mathrm{mM}$ glycerol, for mixotrophic cultivation. For initial screening, strains were cultivated in triplicate in $3.5 \mathrm{ml}$ of BBM with and without $25 \mathrm{mM}$ of glycerol in 12 well plates incubated under continuous light at $40 \mathrm{~W} / \mathrm{m}^{2}$ at room temperature. Growth curves were developed by measuring optical density (OD) at 630nm using an EL-800 universal microplate reader from Bio-Tek instruments, Inc., over ten days. For some experiments, strains were grown in a shaker in $125 \mathrm{ml}$ Erlenmeyer flasks containing $50 \mathrm{~mL}$ of BBM with and without $25 \mathrm{mM}$ glycerol. The strains were grown in constant light at $40 \mathrm{~W} / \mathrm{m}^{2}$ intensity at room temperature and shaken at $160 \mathrm{rpm}$. Each day, $200 \mu \mathrm{l}$ of culture were taken and placed in 96-well microplate for measurement of OD at 630nm as indicated above 
for the 12 well plates. For all experiments pre-inocula were grown under photoautotrophic conditions.

\subsection{Biomass and Lipid Quantification}

Biomass (dry weight) was calculated using the relationship $\mathrm{OD}_{630} /$ gm dry wt $=1.055 \pm$ 0.12 previously determined (Abdelaziz et al., 2014). Nile red, a fluorescent dye capable of staining neutral lipids, was used to quantify intracellular lipids ((Bertozzini et al, 2011; Kou et al, 2013) using a slight modification of previously used methods (Abdelaziz et al., 2014; Leite et al., 2015). Algal samples were diluted with BBM to obtain 0.06 OD for each sample and $143 \mu$ was transferred into black flat-bottom 96 well plates. To this, $50 \mu 1$ of DMSO was added followed by the addition of $6.66 \mu \mathrm{l}$ of Nile red solution $(15 \mu \mathrm{g} / \mathrm{ml}$ Nile red in acetone). The plate was incubated on a microplate shaker at room temperature in dim light for 10 minutes. The plates were read with a spectrofluorometer with excitation at $525 \mathrm{~nm}$ and emission at 580nm. A standard curve made with triolein was created each time samples were to be measured.

\subsection{Analytical procedures}

Glycerol was quantified using the colorimetric method described by Bondioli and Bella (2005) as previously modified for use with 96 well plates (Leite et al., 2015). Algal strains were characterised for their fatty acid composition through extraction and conversion to FAMEs (fatty acid methyl esters). A wet lipid extraction protocol was used which extracted and transesterified the TAGs (triacylglycerols) in a single step. $0.5 \mathrm{ml}$ of algal culture was placed into $4 \mathrm{ml}$ screw cap vials to which $1 \mathrm{ml}$ of saponification reagent $(5 \mathrm{ml}$ of $0.8 \mathrm{~g} / \mathrm{ml} \mathrm{KOH}, 95 \mathrm{ml}$ methanol) was added. The mixture was vortexed for 20 to 30 seconds, incubated at $100^{\circ} \mathrm{C}$ for 90 minutes in a Bloc heater and then left to cool to room temperature after which $1.5 \mathrm{ml}$ of methylation reagent $(17.5 \mathrm{ml}$ $12 \mathrm{~N} \mathrm{HCl}, 282.5 \mathrm{ml}$ methanol) was added. After the samples had been incubated at $60^{\circ} \mathrm{C}$ overnight 
and cooled to room temperature, $1.25 \mathrm{ml}$ hexane was added and the vials were vortexed for 30 minutes. An aliquot of the upper layer was analysed for FAMEs on an Agilent 5975 gas chromatograph coupled with a mass spectrophotometer detector (GC-MS) equipped with a 30m Zebron ZB-5 column (Phenomenex) using a 1:20 split injection with helium as carrier $(1 \mathrm{ml} / \mathrm{m})$ and a temperature gradient $\left(150-250^{\circ} \mathrm{C}\right.$ over $\left.15 \mathrm{~min}\right)$.

\subsection{Growth Rate, Percent Lipid and Lipid Productivity}

Growth rates were calculated according to the standard formula:

$$
\mu=\frac{\ln (\mathrm{Xt} 1)-\ln (\mathrm{Xt} 2)}{\mathrm{t} 1-\mathrm{t} 2} \quad \text { (Eq. 1) }
$$

Where $\mu$ is the growth rate in days ${ }^{-1}, X_{t 1}$ and $X_{t 2}$ are the optical densities at time $t 1$ and $t 2$. For maximum growth rates, $\mathrm{X}_{\mathrm{t} 1}$ and $\mathrm{X}_{\mathrm{t} 2}$ were taken as the point at which cultures enter and complete exponential growth. Thus, t1 was the experimental time point where a specific culture had entered exponential growth and $\mathrm{t} 2$ was the experimental time point where a specific culture had completed exponential growth. As evident from the growth curves, these had to be individually determined for each strain.

Lipid content was calculated as the ratio of the total amount of lipids produced to the total amount of biomass produced by a culture:

Lipid percentage $=\frac{\text { Total amount of lipids produced }(\text { in } m g)}{\text { biomass }(\text { in } m g)}$ (Eq. 2)

Lipid productivity is defined as the lipid yield per volume of growth medium per unit time and is often expressed as $\mathrm{mg} / \mathrm{L} / \mathrm{hour}$ or $\mathrm{mg} / \mathrm{L} / \mathrm{Day}$. Thus, to calculate lipid productivity the following formula was used:

Lipid Productivity $=\frac{\text { Total lipid }(\mathrm{mg})}{(\text { volume of growth media }(\mathrm{L})) *(\text { days of growth })}$ (Eq. 3) 


\subsection{Phylogenetic Analysis}

Algal strains were identified using phylogenetic analysis. Each strain was sequenced for the $18 S r R N A$ gene and for the $r b c L$ gene (Rubisco large subunit). The $18 S r R N A$ and $r b c L$ genes are common markers used for phylogenetic studies due to the presence of highly conserved regions within the sequences of these genes. Genomic DNA was extracted using a modified protocol (Fawley et al, 2004). A volume of one to $2 \mathrm{ml}$ of algal culture was centrifuged at maximum speed for 1 minute. The supernatant was discarded and $200 \mu$ l of extraction buffer (1M $\mathrm{NaCl}, 70 \mathrm{mM}$ Tris, 30mM Na 2 EDTA, $\mathrm{pH}$ 8.6) was added followed by brief vortexing. This mixture was centrifuged to pellet cells again and the supernatant was discarded. To the pellet $200 \mu l$ of fresh extraction buffer was added, along with a quantity of glass beads to fill the conical part of the centrifuge tube, and $25 \mu \mathrm{l}$ of $10 \%$ DTAB and $200 \mu \mathrm{l}$ of chloroform were added. The tubes were vortexed for 20 seconds at the highest speed and the mixture was centrifuged at $2000 g$ for two minutes. After phase separation, $100 \mu \mathrm{l}$ upper aqueous phase was taken and added to a $1.5 \mathrm{ml}$ microtube. The genomic DNA was then purified using a Gel/ PCR DNA Fragments Extraction kit from Geneaid (Lot No. JL33414).

Polymerase chain reactions (PCR) were conducted with the genomic DNA using $18 S$ rRNA internal universal primers and $r b c L$ internal universal primers:

18S Forward primer 5'-GTGGTAACGGGTGACGG-3' and 18S reverse primer 5'GTGCGGCCCAGAACATC-3'

$r b c L$ forward primer 5'-CTCCTCAACCAGGTGTTCC-3' and $r b c L$ reverse primer 5'CTGGCATGTGCCATACGTG-3'. The PCRs were performed with a Phusion High fidelity DNA Polymerase kit from Finnzymes using the protocol supplied by the manufacturer. PCR products were electrophoresed through $0.8 \%$ agarose gels and the PCR products purified as above. Purified PCR products were sequenced at IRIC (Institut de Recherche en Immunologie et 
Cancérologie) affiliated with the University of Montreal. The sequences obtained were aligned with different $18 S r R N A$ and $r b c L$ sequences using BLAST database and the NCBI database to find the closest related organisms. Subsequently, the sequences of the algal strains were aligned with nearest neighbour sequences and outlier sequences using MEGA (Tamura et al, 2011). MUSCLE was used to align sequences after which neighbour joining (N-J) tree were created using the Jukes-Cantor model at a 1000 bootstraps. The phylogenetic relationships of the algal strains were modelled after the algal classification shown elsewhere (Guiry et al, 2014).

\section{Results and discussion}

3.1 Screening strains for enhanced growth and biomass production in the presence of glycerol. Growth rates and lipid production of twelve algal strains were compared under mixotrophic and photoautotrophic conditions in order to identify strains capable of enhanced growth and/or lipid production in the presence of glycerol (Table 1). Measurement of glycerol consumption showed that all strains were capable of taking up glycerol from the growth medium. The percent glycerol removed by the strains was: $\mathrm{PCH} 02,21.0 \pm 5.8$; $\mathrm{PCH} 05,28.9 \pm 10.1 ; \mathrm{PCH} 11,25.4 \pm 4.45 ; \mathrm{PCH} 20$,

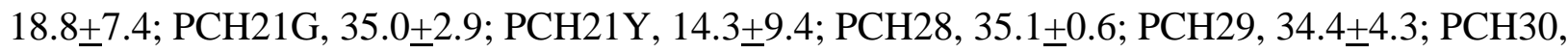
61.2 $\pm 1.07 ; \mathrm{PCH} 32,30.6 \pm 0.77 ; \mathrm{MA} 1 \mathrm{~A} 08,27.0 \pm 4.3 ; \mathrm{MA} 1 \mathrm{~A} 21,34.1 \pm 5.7$. Maximum growth rates ( $\mu$ (day-1)) were determined by measuring growth daily (Figure S1) under both mixotrophic and photoautotrophic growth conditions and volumetric biomass production under the two conditions was determined after ten days of growth at which point most of the algal strains examined had entered stationary phase. With either the calculated maximum growth rates (Table 1) or the individual growth curves (Figure S1) it can be seen that various responses to the presence of glycerol are present. 
There was an appreciable variation in maximum growth rate observed under mixotrophic conditions depending upon the strain (Table 1), with some, PCH05, PCH21Y, and PCH32, showing quite high specific growth rates (from 0.283 to 0.372 day $^{-1}$ ), whereas others, MA1A08 and MA1A21, gave appreciably lower maximum growth rates $\left(0.105\right.$ and $\left.0.141 \mathrm{day}^{-1}\right)$. Regardless of other factors, this measure would be important in choosing strains to develop for a practical production system. To directly examine the effect of glycerol addition on each strain, the fold increase in specific growth rate under mixotrophic conditions compared to photoautotrophic conditions was determined (Table 1). By this measure, four strains, $\mathrm{PCH} 02$, PCH05, PCH32 and MA1A21showed greatly enhanced growth rates in the presence of glycerol (1.53 to 2.44 fold), the specific growth rates of five strains, $\mathrm{PCH} 11$, $\mathrm{PCH} 20, \mathrm{PCH} 21 \mathrm{Y}, \mathrm{PCH} 28$, and MA1A08, were relatively unaffected by glycerol addition, and the growth of three strains, PCH21G, PCH29 and PCH 30, appeared to be inhibited when glycerol was present with a $18 \%$ to $49 \%$ reduction in specific growth rates. The decrease in growth rate of strains $\mathrm{PCH} 21 \mathrm{G}, \mathrm{PCH} 29$, and $\mathrm{PCH} 30$ in the presence of glycerol is reminiscent of a previous study which demonstrated that addition of glycerol inhibited the growth of Chlorella vulgaris (Heredia-Arroyo et al., 2011). On the other hand, the significant increase in growth rate observed with strains $\mathrm{PCH} 02$, $\mathrm{PCH} 05$, PCH32 and MA1A21 in the presence of glycerol mirrors the effects previously noted in other studies where the addition of improved the growth of Chlorella by 18\% (Skorupskaite et al., 2015) or the biomass productivity of Chlorella sorokiniana (Park et al., 2012).

There are probably several possible explanations for the different effects of the presence of glycerol on the maximum growth rate of the various strains. However, without a doubt, carbon assimilation and partitioning must play important roles. If one assumes that the rate of carbon fixation, which indirectly depends upon the rate of photosynthesis, is the major growth limiting factor, then glycerol assimilation would help to overcome this bottleneck, thus possibly 
explaining the increased growth rates in strains where this was observed. Strains where the presence of glycerol was without effect on growth rate could in fact be incapable of assimilating this compound. However, analysis of glycerol concentrations showed that it was consumed (the percent glycerol removed by the strains was: $\mathrm{PCH} 02, \quad 21.0 \pm 5.8 ; \mathrm{PCH} 05,28.9 \pm 10.1 ; \mathrm{PCH} 11$,

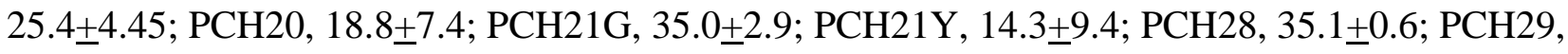
34.4 $\pm 4.3 ; \mathrm{PCH} 30,61.2 \pm 1.07 ; \mathrm{PCH} 32,30.6 \pm 0.77 ; \mathrm{MA} 1 \mathrm{~A} 08,27.0 \pm 4.3 ; \mathrm{MA} 1 \mathrm{~A} 21,34.1 \pm 5.7$.$) ,$ suggesting that it might have been assimilated but converted into materials, such as storage compounds, that do not directly lead to cell growth. Finally, two strains actually grew slower in the presence of glycerol, suggesting that in some cases this compound could act as an inhibitor, possibly by acting to unbalance metabolism after its assimilation.

Some of these aspects were investigated further. In terms of the effect of the presence of glycerol on biomass production, again, the strains could be divided into three different groups. Five strains, $\mathrm{PCH} 02, \mathrm{PCH} 11, \mathrm{PCH} 20, \mathrm{PCH} 29$, and MA1A21 had final biomass yields under mixotrophic conditions that were higher (1.12 to 1.92 fold) higher than under photoautotrophic conditions. Similar results have been noted in some previous studies with Neochloris oleabundans, Botryococcus braunii and Dunaliella sp. (Choi and Lee 2015) or with various strains of Chlorella (Leite et al., 2015; Liang et al. 2009; Lin and Wu, 2015; Park et al., 2012; Skorupskaite et al., 2015). One strain, PCH30, showed a lower amount ( 0.86 fold) of final biomass in the presence of glycerol (mixotrophic conditions) and finally, the biomass yields of six strains, $\mathrm{PCH} 05, \mathrm{PCH} 21 \mathrm{G}, \mathrm{PCH} 21 \mathrm{Y}, \mathrm{PCH} 28, \mathrm{PCH} 32$, and MA1A08, were indifferent to the presence of glycerol, differing by less than $10 \%$ under the two different conditions.

Two different mechanisms might be at play to produce higher biomass under mixotrophic conditions. In one scenario, assimilated glycerol could be primarily channelled into storage molecule production, such as lipids or sugars, and consequently, algal cells would be "fattened 
up". In a second scenario, assimilated glycerol could be directed into synthesis of essential cell components, thus directly working to increase the total number of cells observed at the end of growth. Similarly to what was observed with maximum growth rates, total biomass production varied widely with the different strains. By the time they had reached stationary phase, almost all strains had produced more than $0.5 \mathrm{~g} / \mathrm{L}$ biomass, with strain $\mathrm{PCH} 29$ showing the highest production under the mixotrophic growth conditions used here $(1.02 \mathrm{~g} / \mathrm{L})$. On the other hand, strains MA1A08 and MA1A21 were the lowest biomass producers, giving only $0.16 \mathrm{~g} / \mathrm{L}$ and $0.497 \mathrm{~g} / \mathrm{L}$. Thus, although, strain MA1A21 had a high increase in biomass production under mixotrophic conditions (1.3 fold), its final biomass production is relatively low and consequently this strain would not be very suitable for mass cultivation.

Interestingly enough, an increased maximum growth rate under mixotrophic conditions does not necessarily correlate with increased biomass production under the same conditions (Table 1), i.e. strains with high growth rates under mixotrophic conditions do not necessarily show higher biomass production. For example, $\mathrm{PCH} 05$ had a $62 \%$ higher growth rate under mixotrophic conditions, but had similar biomass production under both mixotrophic and photoautotrophic conditions. Similarly, PCH02 and MA1A21 were the only strains to have both significantly high biomass and high maximum growth rates under mixotrophic conditions. When cultivated with glycerol, PCH02 showed a 1.92 fold increase in biomass and a 1.53 fold increase in growth rate, and MA1A21 had a 1.30 fold increase in biomass production and a 2.44 fold increase in growth rate. Consequently, these findings suggest that with these strains glycerol played a significant role in biomass increase, either through increased cell production or through increased production of storage molecules, such as lipids.

Thus, glycerol seemed to have different effects on biomass production and growth rates. Two strains, PCH02 and MA1A21, showed increased growth rates and biomass when grown with 
glycerol. However, MA1A21 presented one of the lowest growth rates and biomass production. These results show that, at least with some strains, glycerol addition can be used to greatly increase growth rates and biomass production. This is a significant finding which suggests that, depending upon the strain, the use of glycerol would be of great benefit in increasing both growth rates and biomass production. Hence, it was of interest to determine if glycerol addition could enhance lipid production.

\subsection{Effect of glycerol on lipid production}

When the amount of lipid produced as a percentage of total dry weight is examined for the strains grown under both mixotrophic and photoautotrophic conditions it can be seen that more than half of the strains (eight; PCH02, PCH05, PCH11, PCH20, PCH29, PCH30, MA1A08 and MA1A21)) had a significantly higher lipid content when grown under mixotrophic conditions (Figure 1A). While most strains showed increases that were 30 to $50 \%$ above the content seen under photoautotrophic conditions, two, MA108 and PCH02, doubled and tripled their lipid content under mixotrophic conditions. Since the only difference in the two growth conditions was the presence of glycerol, this suggests that these algal strains largely utilise the assimilated glycerol for lipid production. At any rate, these results show that for the majority of strains examined, lipid content is very significantly enhanced by mixotrophic growth on glycerol. Moreover, in the presence of glycerol, and without other, additional, measures taken to increase lipid production, six strains, $\mathrm{PCH} 02$, $\mathrm{PCH} 05, \mathrm{PCH} 11, \mathrm{PCH} 20, \mathrm{PCH} 29$ and MA1A21 showed a lipid content of $20 \%$ or greater, making them potentially interesting strains for further development for biofuels production.

Of course, for practical biofuels production, the volumetric lipid production, a function of both percent lipid content and total biomass production, would be a very important consideration. Some interesting observations in this regard can be made when this is examined with these strains 
(Figure 1B). For one thing, total lipid production was highly variable, differing greatly between the various strains. Some produced only minimal amounts of neutral lipids under all conditions and thus can be eliminated from consideration for biofuels production. From this point of view, six strains were of some interest since they produced at least $0.1 \mathrm{~g} / \mathrm{L}$ neutral lipid and three strains, $\mathrm{PCH} 05, \mathrm{PCH} 20$ and $\mathrm{PCH} 29$, might be of particular interest in that they produced more than $0.2 \mathrm{~g} / \mathrm{L}$ neutral lipids. Total lipid production in these strains was $40 \%$ to $60 \%$ higher under mixotrophic conditions. It should be pointed out that these levels of production were obtained without special measures, e.g. nitrogen deprivation, being taken to enhance lipid production. Another strain of potential interest is $\mathrm{PCH} 02$, which, although having only a modest level of total lipid production $(0.14 \mathrm{~g} / \mathrm{L}$ (mixotrophic)), showed a very remarkable nearly six-fold increase in the presence of glycerol. Thus, the addition of glycerol can, with the proper strain, greatly increase total lipid production, of potential great significance in the further development of algal biofuels.

\subsection{GC-MS and flow cytometry analysis of lipid production}

In addition to quantity, the type of lipid produced is very important in terms of the fuel characteristics of the resultant biodiesel. A partial survey of some of the strains in this study, including three of the top producers, PCH05, PCH11, PCH20 (Table 2), was made using a wetlipid extraction transesterification procedure with analysis of the FAME profile using GC-FID. In general all strains showed high percentages of FAMES derived from long chain fully saturated or mono-unsaturated fatty acids, palmitic (16:0), oleic (18:1) and steric (18:0), which give a biodiesel with good fuel properties (Hoekman et al., 2012). There was some variation in the composition depending upon whether the culture was grown with glycerol or not. Interestingly, two strains, PCH05 and PCH21Y, produced small but significant amounts of the nutritionally important unsaturated PUFA (polyunsaturated fatty acid) linoleic acid. 
Flow cytometry was used to examine the lipid and chlorophyll contents of the different strains that had been incubated under photoautotrophic and mixotrophic conditions (Table 3). This technique, although relatively little used in this area of research, can give highly valuable information on the level of the individual cell as well as showing the population distribution of a particular trait. Chlorophyll is naturally fluorescent and the mean chlorophyll content per cell can be read out using the FL3 channel. The neutral lipid content per cell can be assessed by using the fluorescence read out of the FL1 channel of Bodipy ${ }^{\circledR}$-stained cells. This analysis showed that different strains responded differently in terms of both their chlorophyll content and lipid content when incubated in medium containing glycerol (Figure 2 and Table 3). Some strains, PCH02 (Figure 2A), PCH29, and MA1A2, had significantly higher lipid content per cell (1.36-1.52 fold) when glycerol was present, whereas others, strains PCH05 (Figure S2B), PCH11, PCH21G, PCH21Y, PCH28, PCH30, PCH32, MA1A08, were either relatively indifferent in this regard, or showed a significant decrease in lipid content (PCH20) (Table 3). Two of the strains showing a significant increase in neutral lipids in the presence of glycerol also showed a significant decrease in chlorophyll content: PCH02 (Figure 2A), 1.85 fold; MA1A21, 2.13 fold. These results show that the entire algal population in this case is shifted into a state with high lipid content. On the other hand, a strain which gave significantly higher lipid levels in the presence of glycerol, strain PCH29, showed, like most of the other strains, very little difference in chlorophyll content between the presence and absence of glycerol. Interestingly, one strain, PCH05, was unusual in that it showed higher chlorophyll content (1.3 fold) in the presence of glycerol (Figure $2 \mathrm{~B})$.

\subsection{Phylogenetic analysis}

Since the strains examined showed great differences in their growth and lipid production under photoautotrophic conditions and in their response to the presence of glycerol, it was of interest to determine their phylogenetic relatedness to each other and to previously characterized 
strains. Genomic regions of each strain corresponding to the 18S rRNA and RUBISCO genes were amplified, sequenced, and phylogenetic trees constructed using the MEGA program. Phylogenetic analysis using the determined $18 \mathrm{~S}$ rRNA sequences shows that all the strains examined belong to the Chlorophyta phylum, also known as the green algae (Figure 3). This analysis shows that $\mathrm{PCH} 05, \mathrm{PCH} 11, \mathrm{PCH} 20$ and $\mathrm{PCH} 29$ are all similar and closely related to Chlorella vulgaris a finding that would appear to agree with the lipid, biomass and growth data, since all of these strains showed similar results. PCH02, which had appeared different from the four strains closely related to $C$. vulgaris in that it showed a very large difference in lipid accumulation under the two conditions, is also a member of the Chlorella genus. However, it was more closely associated to Chlorella sorokiniana. PCH28 belongs to the Hindakia genus, being most closely related to Hindakia fallax. The Hindakia and Chlorella genera are both members of Chlorellaceae. Consequently, an outlier group was created in the tree, which shared a common ancestor with the Chlorella and Hindakia genera. The outlier group contained the Prasiola and Lobosphaera genera. These two genera along with the Chlorella and Hindakia genera all belong to Trebouxiophyceae class in the Chlorophyta phylum. Three strains, PCH21Y, PCH21G and PCH30, were shown to be relatively poor lipid producers (Figure 1B) and the phylogenetic analysis showed them to be very closely related to each other as well as being closely related to Acutodesmus obliquus, which belongs to the Scenedesmaceae family of the Chlorophyta phylum. Likewise, strain PCH32 belongs to the related Scenedesmus genus and is most closely related to $S$. abundans. The partial sequence of the RUBSICO gene of strain suggests that MA1A08 is closely related to the Desmodesmus genus (results not shown) and thus the most related to strain PCH32 amongst the strains examined here.

The strain MA1A21 is most closely related in this analysis to Mychonastes rotundus. The Mychonastes and Monoraphidium genera represent an outlier group to the Scenedesmaceae 
family, with Mychonastes belonging to the Mychonastaceae family and Monoraphidium belonging to the Selenastraceae family, all of which belong to the Sphaeropleales order. Since the Sphaeropleales order belongs to the Chlorophyceae class, it is an outlier group to the Trebouxiophyceae class, which contains PCH02, PCH05, PCH11, PCH20 and PCH28, and thus MA1A21, PCH32, PCH30, PCH21G and PCH21Y are quite different phylogenetically from them.

\section{Conclusion}

Glycerol proved to be a useable carbon-source for mixotrophic growth for most of the algal strains examined here, shown by phylogenetic analysis to be highly diverse within the Chlorophyta phylum. In particular, this demonstrated that even among strains identified as Chlorella there is a wide variation in the response to glycerol, thus explaining some of the seeming contradiction between previous studies. Furthermore, strains PCH02, PCH05, PCH11, PCH20 and PCH29, all within the Chlorella genus, seem to demonstrate the most promise for industrial cultivation due to their high biomass production, high growth rates and high lipid production under mixotrophic conditions.

\section{Acknowledgments}

This research was supported by a grant from FQRNT (Le Fonds Québécois de la recherche sur la nature et les technologies), Programme de recherche en partenariat contribuant à la séquestration des gaz à effet de serre (2011-GZ-141307) to P.C.H. PCH holds a National Research Council Senior Research Associateship Award at the Life Sciences Research Center, Department of Biology, United States Air Force Academy. The views expressed in this article are those of the authors and do not reflect the official policy or position of the United States Air Force, the Department of Defense, or the US Government. 


\section{References}

1. Abdelaziz, A. E. M, Leite, G. B., Hallenbeck, P. C. 2013a. Addressing the challenges for sustainable production of algal biofuels: I. Algal strains and nutrient supply. Environ. Technol. 34, 1783-1805.

2. Abdelaziz, A. E. M, Leite, G. B., Hallenbeck, P. C. 2013b. Addressing the challenges for sustainable production of algal biofuels: II. Harvesting and conversion to biofuels. Environ. Technol. 34, 1783-1805.

3. Abdelaziz, A. E. M, Leite, G. B., Belhaj, M.A., Hallenbeck, P.C. 2014. Screening microalgae native to Quebec for wastewater treatment and biodiesel production, Bioresour.Technol. 157, $140-148$.

4. Andersen, R. A., Berges, J. A., Harrison, P. J., Watanabe, M. M. 2005. Appendix A- Recipes for Freshwater and Seawater Media.” Algal culture techniques. ED. Andersen, R. A. Burlington, USA: Elsevier Academic Press, pp. 429-539.

5. Andruleviciute, V., Makareviciene, V., Skorupskaite, V., Gumbyte, M. 2014. Biomass and oil content of Chlorella sp., Haematococcus sp., Nannochloris sp. and Scenedesmus sp. under mixotrophic growth conditions in the presence of technical glycerol. J.Appl. Phycol. 26, 8390.

6. Bertozzini, E., Galluzzi, L., Penna, A., Magnani, M. 2011. Application of standard addition method for the absolute quantification of neutral lipids in microalgae using Nile Red. J. Microbiol. Methods. 87, 17-23.

7. Bondioli, P., Della Bella, L. 2005. An alternative spectrophotometric method for the determination of free glycerol in biodiesel. Eur. J of Lipid Sci. 107, 153-157. 
8. Chen, Y., Walker, T. H. 2011. Biomass and lipid production of heterotrophic microalgae Chlorella protothecoides by using biodiesel derived crude glycerol. Biotechnol. Lett. 33, 1973-1983.

9. Chisti, Y. 2013. Constraints to commercialization of algal fuels. J.Biotechnol. 167, 201-214.

10. Dubey, K., Kumar, S., Dixit, D., Kumar, P., Kumar, D., Jawed, A., Haque, S. 2015. Implication of Industrial Waste for Biomass and Lipid Production in Chlorella minutissima Under Autotrophic, Heterotrophic, and Mixotrophic Grown Conditions. Appl. Biochem. Biotechnol., 176, 1581-1595.

11. Fawley, M. W., Fawley, K. P. 2004. A simple and Rapid Technique for the isolation of DNA from Microalgae. J. Phycol. 40, 223-225.

12. Guiry, M. D., Guiry, G. M. “AlgaeBase.” World-wide electronic publication, National University of Ireland, Galway. http://www.algaebase.org; accessed July 2014.

13. Hallenbeck, P.C., Leite, G.B., Abdelaziz, A.E.M., 2014. Exploring the diversity of microalgal physiology for applications in wastewater treatment and biofuel production. Algal Res. 6, $111-118$.

14. Heredia-Arroyo, T., Wei, W., Ruan, R., Hu, B. 2011. Mixotrophic cultivation of Chlorella vulgaris and its potential application for the oil accumulation from non-sugar materials. Biomass Bioenergy. 35, 2245-2253.

15. Hoekman, S. K., Broch, A., Robbins, C., Ceniceros, E., Natarajan, M. 2012. Review of biodiesel composition, properties, and specifications. Ren. Sust. Energy Rev.. 16, 143-169.

16. Koller, M., Salerno, A., Tuffner, P., Koinigg, M., Böchzelt, H., Schober, S., Pieber, S., Schnitzer, H., Mittelbach, M., Braunegg. G. 2012. Characteristics and potential of micro algal cultivation strategies: a review. J. Clean. Prod. 37, 377-388 
17. Kou, Z., Bei, S., Sun, J., Pan, J. 2013. Fluorescent measurement of lipid content in the model organism Chlamydomonas reinhardtii. J. Appl. Phycol. 25, 1633-1641.

18. Leite, G. B., Hallenbeck, P. C. “Algae Oil.” Microbial Technologies in Advanced Biofuels Production. Ed. Hallenbeck, P. C. New York, New York, USA: Springer Science + Business Media, 2012. 231-259.

19. Leite, G. B., Paranjape, K., Abdelaziz, A. E. M., Hallenbeck, P. C. 2015. Utilization of biodiesel-derived glycerol or xylose for increased growth and lipid production by indigenous microalgae. Bioresour Technol 184, 123-130

20. Liang, Y., Sarkany, N., Cui, Yi. 2009. Biomass and Lipid productivities of Chlorella vulgaris under autotrophic, heterotrophic and mixotrophic growth conditions. Biotechnol. Lett. 31, 1043-1049.

21. Lin, T.-S., Wu, J.-Y. 2015. Effect of carbon sources on growth and lipid accumulation of newly isolated microalgae cultured under mixotrophic condition. Bioresour. Technol. 184, 100-107.

22. Lowrey, J., Brooks, M., McGinn, P. 2015. Heterotrophic and mixotrophic cultivation of microalgae for biodiesel production in agricultural wastewaters and associated challenges — a critical review. J. Appl. Phycol. 27, 1485-1498.

23. Miazek K, Remacle C, Richel A, Goffin D. 2014. Effect of lignocellulosic related compounds on microalgae growth and product biosynthesis: A review. Energies 7, 4446-4481.

24. Mitra, D., van Leeuwen, J., Lamsal, B. 2012. Heterotrophic/mixotrophic cultivation of oleaginous Chlorella vulgaris on industrial co-products. Algal Res. 1, 40-48.

25. Mohan, S.V., Rohit, M.V., Chiranjeevi, P., Chandra, R., Navaneeth, B. 2015. Heterotrophic microalgae cultivation to synergize biodiesel production with waste remediation: Progress and perspectives. Bioresour. Technol. 184, 169-178. 
26. Muto, M., Tanaka, M., Liang, Y., Yoshino, T., Matsumoto, M., Tanaka, T. 2015.

Enhancement of glycerol metabolism in the oleaginous marine diatom Fistulifera solaris JPCC DA0580 to improve triacylglycerol productivity. Biotechnol. Biofuels, 8, 1-7.

27. Ogawa, T., Aiba, S. 1981. Bioenergetic Analysis of Mixotrophic Growth in Chlorella vulgaris and Scenedesmus auctus. Biotechnol. Bioeng.. 23, 1121-1132.

28. O’Grady, J., Morgan, J. A. 2011. Heterotrophic growth and lipid production of Chlorella protothecoides on glycerol. Bioprocess Biosystems Eng. 34, 121-125.

29. Pancha, I., Chokshi, K., Mishra, S. 2015. Enhanced biofuel production potential with nutritional stress amelioration through optimization of carbon source and light intensity in Scenedesmus sp CCNM 1077. Bioresour. Technol. 179, 565-572

30. Park, K., Whitney, C., McNichol, J., Dickinson, K., MacQuarrie, S., Skrupski, B., Zou, J., Wilson, K., O’Leary, S.B., McGinn, P. 2012. Mixotrophic and photoautotrophic cultivation of 14 microalgae isolates from Saskatchewan, Canada: potential applications for wastewater remediation for biofuel production. J.Appl. Phycol. 24, 339-348.

31. Skorupskaite, V., Makareviciene, V., Levisauskas, D. 2015. Optimization of mixotrophic cultivation of microalgae Chlorella sp for biofuel production using response surface methodology. Algal Res.-Biomass Biofuels Biopro. 7, 45-50.

32. Souza, S.P., Gopal, A.R., Seabra, J.E.A. 2015. Life cycle assessment of biofuels from an integrated Brazilian algae-sugarcane biorefinery. Energy, 81, 373-381.

33. Tamura, K., Peterson, D., Peterson, N., Stecher, G., Nei, M., Kumar, S. 2011. MEGA5: Molecular Evolutionary Genetics Analysis using Maximum Likelihood, Evolutionary Distance, and Maximum Parsimony Methods. Mol. Biol. Evol. 28, 2731-2739.

34. Wang, J., Yang, H., Wang, F. 2014. Mixotrophic Cultivation of Microalgae for Biodiesel Production: Status and Prospects. Appl. Biochem. Biotechnol. 172, 3307-3329 
35. Yen, H.-W., Chang, J.-T. 2013. A two-stage cultivation process for the growth enhancement of Chlorella vulgaris. Biopro. Biosyst. Eng. 36, 1797-1801. 


\title{
Figure legends
}

Figure 1: The effect of glycerol on the percent lipid and total lipid production of the twelve strains examined

\author{
A) Lipid percentage by dry weight for each algal strain grown under mixotrophic conditions, with \\ $25 \mathrm{mM}$ of glycerol, and photoautotrophic conditions for ten days under continuous light at \\ 40W/m2 at room temperature. B) Volumetric lipid production (g/L) for each algal strain grown \\ under mixotrophic conditions, with $25 \mathrm{mM}$ of glycerol, and photoautotrophic conditions for ten \\ days under continuous light at $40 \mathrm{~W} / \mathrm{m} 2$ at room temperature.
}

Figure 2: FACS analysis of changes in cell size and cellular lipid content for two strains

Figure 3: Neighbour joining tree showing phylogenetic relationship between each algal strains. This phylogenetic tree is a neighbour joining tree using Jukes-Cantor model with 1000 bootstraps. Each number on the tree represents the bootstrapping values. Bootstrapping is a resampling method and helps to assign measure of accuracy to sample estimates. The values on the trees are the bootstrapping values and thus represent the accuracy percentages 


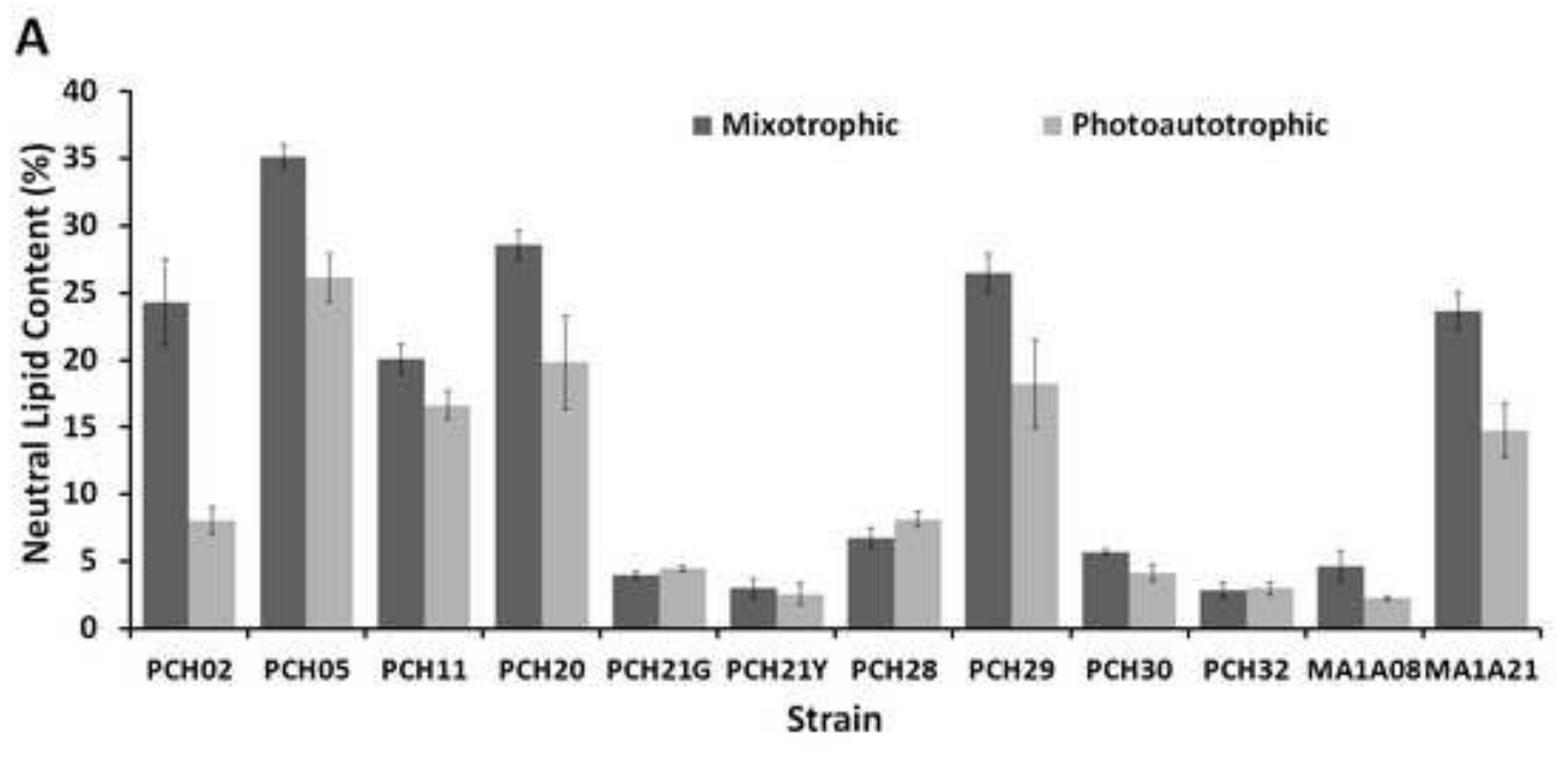

B

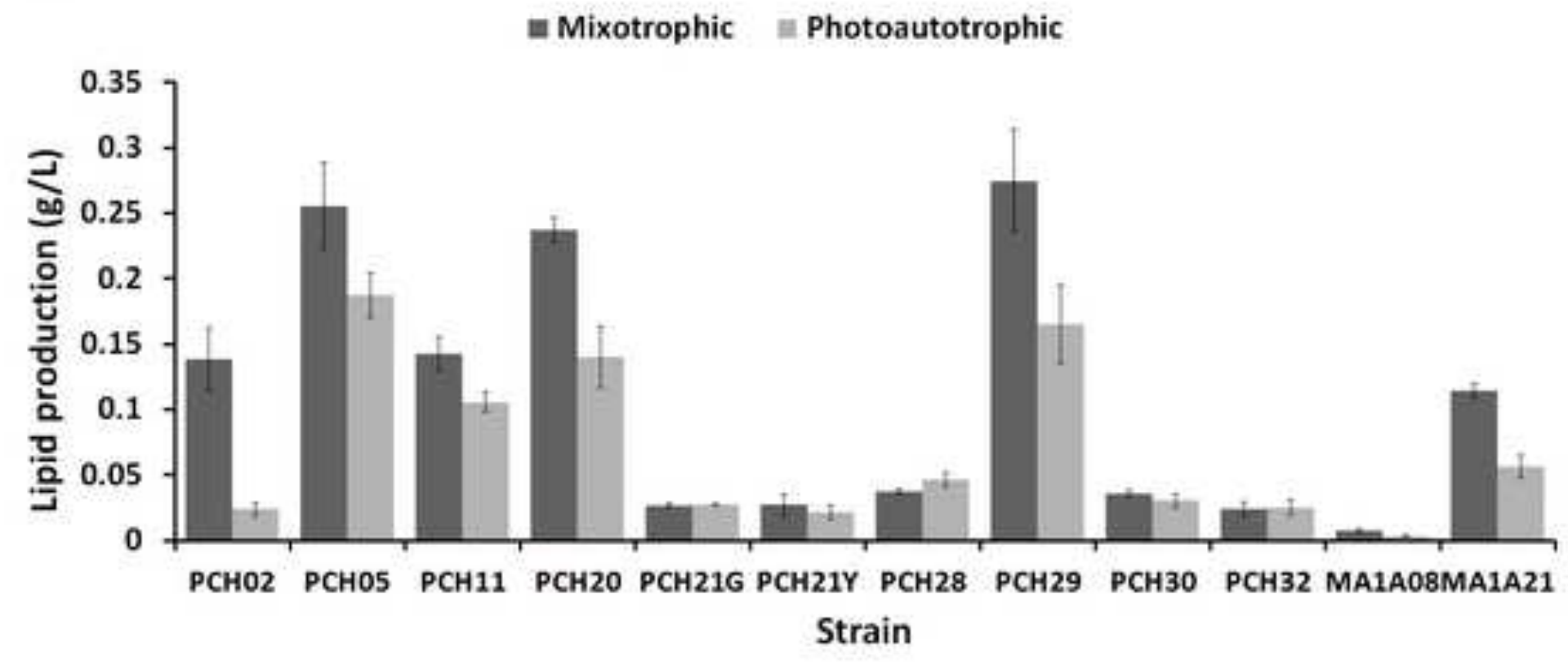



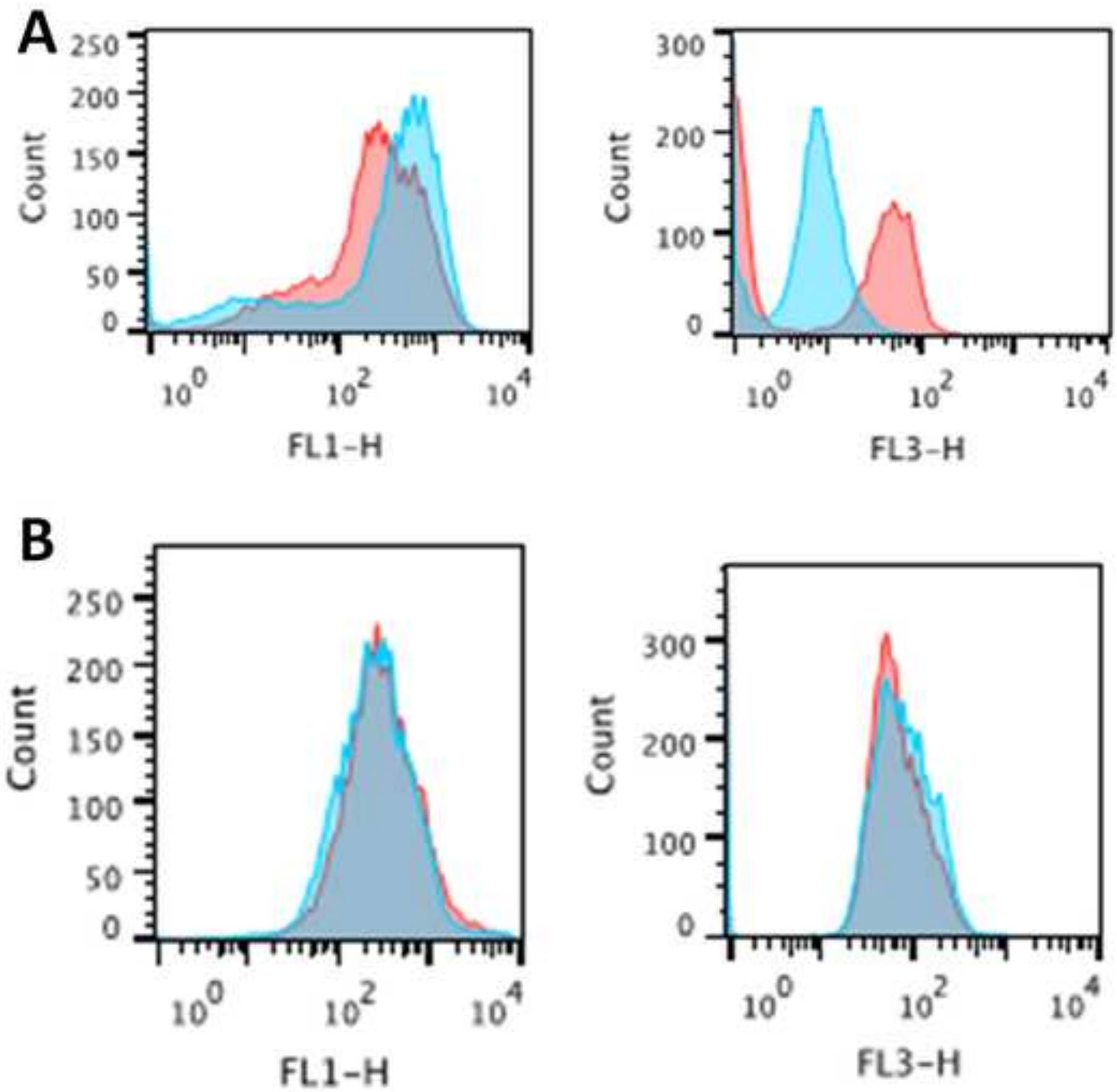


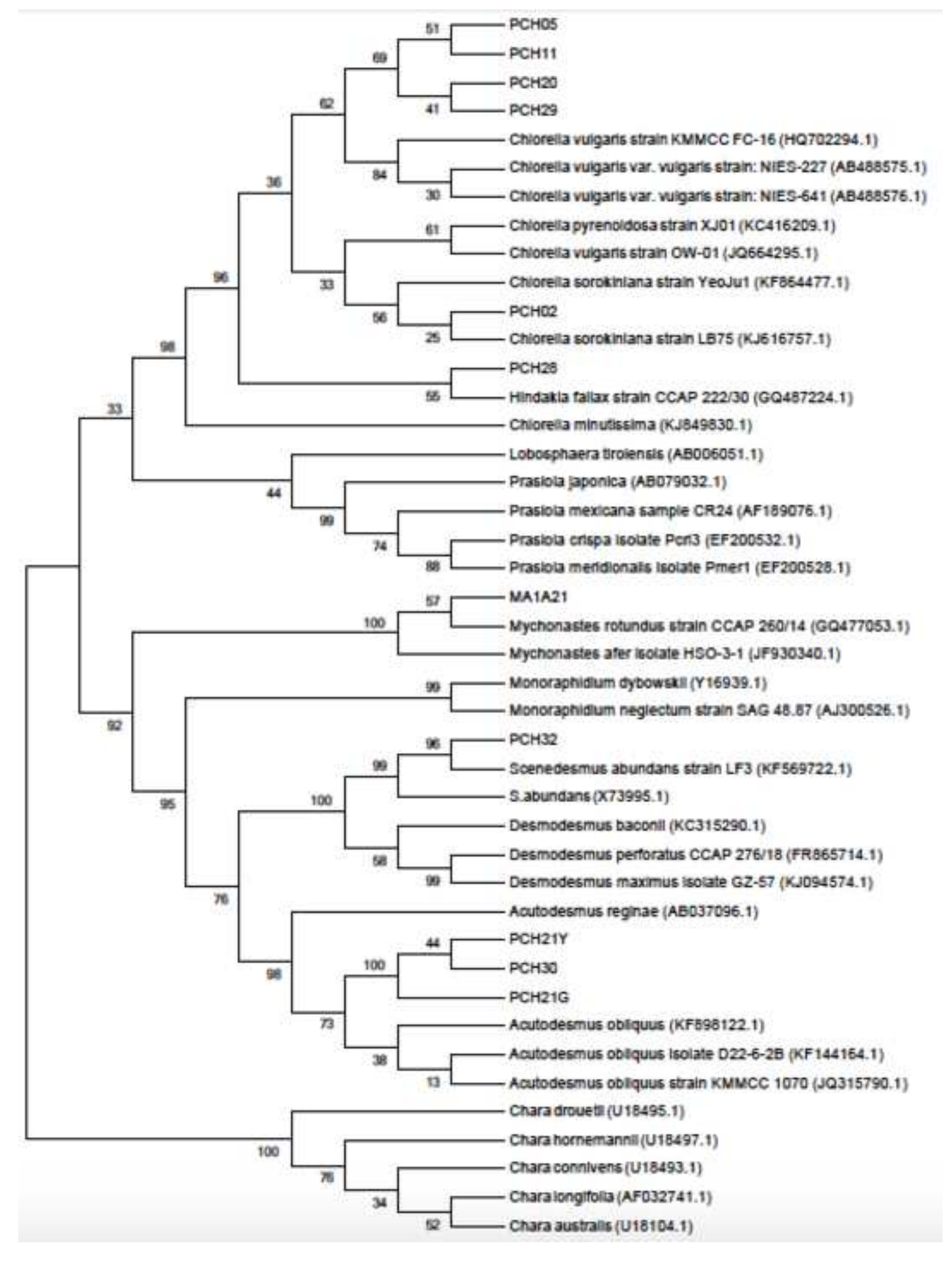

.


Table 1: Final biomass and maximum specific growth rates of the strains under mixotrophic and photoautotrophic conditions

\begin{tabular}{|c|c|c|c|c|c|c|}
\hline \multirow{2}{*}{$\begin{array}{l}\text { Algal } \\
\text { Strain }\end{array}$} & \multicolumn{3}{|c|}{ Biomass $\left(\mathrm{g} \mathrm{L}^{-1}\right)$} & \multicolumn{3}{|c|}{ Growth rate $\left(\right.$ day $\left.^{-1}\right)$} \\
\hline & Mixotrophic & Photoautotrophic & Fold & Mixotrophic & Photoautotrophic & Fold \\
\hline PCHO2 & $0.57 \pm 0.033$ & $0.30+0.040$ & 1.92 & $0.26 \pm 0.037$ & $0.17 \pm 0.024$ & 1.53 \\
\hline PCHO5 & $0.73 \pm 0.078$ & $0.72 \pm 0.076$ & 1.01 & $0.28 \pm 0.032$ & $0.18 \pm 0.037$ & 1.62 \\
\hline PCH11 & $0.71 \pm 0.029$ & $0.63 \pm 0.005$ & 1.12 & $0.23 \pm 0.001$ & $0.20 \pm 0.040$ & 1.16 \\
\hline PCH2O & $0.83 \pm 0.006$ & $0.71 \pm 0.040$ & 1.17 & $0.23 \pm 0.006$ & $0.27 \pm 0.074$ & 0.86 \\
\hline PCH21G & $0.68 \pm 0.009$ & $0.62 \pm 0.030$ & 1.08 & $0.26 \pm 0.031$ & $0.51 \pm 0.047$ & 0.51 \\
\hline PCH21Y & $0.90 \pm 0.080$ & $0.86 \pm 0.042$ & 1.05 & $0.37 \pm 0.043$ & $0.32 \pm 0.018$ & 1.16 \\
\hline РCH28 & $0.56 \pm 0.066$ & $0.57 \pm 0.039$ & 0.97 & $0.24 \pm 0.008$ & $0.23 \pm 0.023$ & 1.04 \\
\hline РСH29 & $1.02 \pm 0.080$ & $0.91 \pm 0.045$ & 1.12 & 0.2510 .034 & $0.31 \pm 0.035$ & 0.82 \\
\hline РСH30 & $0.63 \pm 0.025$ & $0.74 \pm 0.024$ & 0.86 & $0.22 \pm 0.025$ & $0.29 \pm 0.039$ & 0.76 \\
\hline РCH32 & $0.85 \pm 0.018$ & $0.84 \pm 0.057$ & 1.01 & $0.37 \pm 0.036$ & $0.24 \pm 0.041$ & 1.55 \\
\hline MA1A08 & $0.16 \pm 0.005$ & $0.15 \pm 0.014$ & 1.06 & $0.11 \pm 0.005$ & $0.10 \pm 0.010$ & 1.06 \\
\hline MA1A21 & $0.50 \pm 0.018$ & $0.38 \pm 0.009$ & 1.30 & $0.14 \pm 0.031$ & $0.06 \pm 0.029$ & 2.44 \\
\hline
\end{tabular}

Cultures were grown in triplicate under mixotrophic $(25 \mathrm{mM}$ glycerol) and photoautotrophic conditions under constant light at room temperature for ten days. 
Table 2: Fatty Acid Methyl Ester (FAME) composition (\%) of several strains grown under both mixotrophic conditions (25mM glycerol) and photoautotrophic conditions

\begin{tabular}{|c|c|c|c|c|c|c|}
\hline \multirow[t]{2}{*}{$\begin{array}{l}\text { Algal } \\
\text { strain }\end{array}$} & \multicolumn{2}{|c|}{$\begin{array}{c}\text { Palmitic acid } \\
(16: 0)\end{array}$} & \multicolumn{2}{|c|}{$\begin{array}{c}\text { Oleic acid } \\
(18: 1)\end{array}$} & \multicolumn{2}{|c|}{$\begin{array}{c}\text { Stearic acid } \\
(18: 0)\end{array}$} \\
\hline & Mixotrophic & Photoautotrophic & Mixotrophic & Photoautotrophic & Mixotrophic & Photoautotrophic \\
\hline $\mathrm{PCH}^{*}{ }^{*}$ & 19 & 28 & 59 & 56 & 18 & 17 \\
\hline PCH11 & 25 & 30 & 52 & 58 & 22 & 12 \\
\hline PCH20 & 29 & 25 & 64 & 67 & 7.2 & 7.8 \\
\hline $\mathrm{PCH} 21 \mathrm{Y}^{*}$ & 34 & 31 & 52 & 58 & 7 & 11 \\
\hline PCH30 & 60 & 45 & 40 & 55 & 0 & 0 \\
\hline PCH32 & 60 & 42 & 22 & 41 & 18 & 16 \\
\hline
\end{tabular}

* The totals with glycerol are less than $100 \%$ since in the presence of glycerol some linoleic acid was found: $\mathrm{PCH} 21 \mathrm{Y}, 7 \%$; $\mathrm{PCH} 05,3 \%$ 
Table 3. Differences in lipid content (mean FL1) and chlorophyll content (mean FL3) of the different strains incubated under mixotrophic and photoautotrophic conditions

\begin{tabular}{lllllll} 
Strain & \multicolumn{3}{c}{ Mean FL1-H } & & & Mean FL3-H \\
& Mixo & Photo & Fold (x) & Mixo & Photo & Fold (x) \\
PCH02 & $550 \pm 171$ & $364 \pm 28$ & 1.52 & $8.9 \pm 5$ & $16.5 \pm 7$ & 0.54 \\
PCH05 & $376 \pm 36$ & $391 \pm 80$ & 0.96 & $92 \pm 3$ & $71.1 \pm 15$ & 1.30 \\
PCH11 & $190+28$ & $176+48$ & 1.08 & $30.5+2.5$ & $34.3+6.9$ & 0.89 \\
PCH20 & $122+4.0$ & $198+126$ & 0.62 & $13.5+2.2$ & $16.9+4.4$ & 0.80 \\
PCH21G & $336+104$ & $384+6.4$ & 0.87 & $13.2+3.3$ & $18.3+1.9$ & 0.72 \\
PCH21Y & $502+71$ & $534+74$ & 0.94 & $9.6+0.1$ & $15.8+1.8$ & 0.61 \\
PCH28 & $461+43$ & $576+37$ & 0.80 & $73.3+3.7$ & $71.6+3.2$ & 1.02 \\
PCH29 & $181+31$ & $130+13$ & 1.39 & $18.3+0.6$ & $21.0+1.7$ & 0.88 \\
PCH30 & $293+25$ & $300+54$ & 0.98 & $24.5+4.8$ & $23.8+4.4$ & 1.03 \\
PCH32 & $301+22$ & $336+13$ & 0.89 & $11+0.7$ & $14.9+0.9$ & 0.74 \\
MA1A08 & $185+33$ & $236+36$ & 0.78 & $31.2+11$ & $41.2+3.2$ & 0.76 \\
MA1A21 & $587+69$ & $431+32$ & 1.36 & $6.9+1.9$ & $14.7+1.0$ & 0.47
\end{tabular}

